nuclear fractionation and western blotting. Activation of the MRN DNA damage response pathway was confirmed by chIP qPCR and western blot analysis.

Results Alizarin red, Alkaline phosphatase activity and RUNX2 and OCN qPCR confirmed development of calcification during $\mathrm{H} 2 \mathrm{O} 2$ treatment, which was reduced with SIRT1 activation. Immunohistochemistry demonstrated enhanced DNA damage marker expression in diabetic vessels compared to non-diabetic control ITA tissue, while SMCs harvested from diabetic patients also showed a significant reduction in SIRT1 expression $(p<0.01)$ and elevated $\mathrm{p} 53$ expression $(\mathrm{p}<0.01)$ compared to cells from non-diabetic patients. SIRT1 expression was reduced in cells following high glucose treatment, in conjunction with increased protein expression of the DNA damage marker, $\gamma \mathrm{H} 2 \mathrm{~A} . \mathrm{X}(\mathrm{p}<0.05)$. The comet assay showed a significant increase in DNA damage in both osteogenic and high glucose conditions following $\mathrm{H} 2 \mathrm{O} 2$ treatment, which also caused an increase in SIRT1 nuclear translocation. Activation of SIRT1 significantly reduced $\mathrm{H} 2 \mathrm{O} 2$ induced DNA damage $(p<0.01)$ and increased recovery after $3 h(p<0.005)$. In addition, deacetylation of MRE11, RAD50 $(\mathrm{p}<0.01)$ and NBS1 $(\mathrm{MRN})(\mathrm{p}<0.005)$ correlated with SIRT1 activation, and an increase in their phosphorylation following $\mathrm{H} 2 \mathrm{O} 2$ treatment.

Conclusions This study demonstrates that SIRT1 protects against $\mathrm{H} 2 \mathrm{O} 2$ induced DNA damage and subsequent calcification within a diabetic environment via activation of the MRN complex. The loss of SIRT1 within the calcified vessels of diabetic patients may contribute to a defective DNA repair mechanism, caused by the absence of SIRT1, resulting in a reduction of MRN deacetylation and thus activation of the DNA damage response, and could be an appropriate target for potential therapeutic intervention for vascular calcification. Conflict of interest N/A

\section{BS60 MOLECULAR MECHANISMS IMPLICATED IN INHIBITION OF ANGIOGENESIS MEDIATED BY THE CALCIUM TRANSPORTER PLASMA MEMBRANE CALCIUM ATPASE 4} ${ }^{3}$ Elizabeth Cartwright, ${ }^{4}$ James Cotton, ${ }^{2}$ Angel L Armesilla. ${ }^{1}$ University of Wolverhampton; ${ }^{2}$ RIHS, FSE, University of Wolverhampton; ${ }^{3}$ Division of Cardiovascular Sciences, University of Manchester, Manchester, UK; ${ }^{4}$ Department of Cardiology, Heart and Lung Centre, New Cross Hospital, Wolverhampton, UK

\subsection{6/heartjn--2019-BCS.220}

Introduction Ischaemic heart disease is a leading cause of death worldwide. Myocardial Infarction (MI) is caused by insufficient or no supply of oxygen to the heart due to narrowing of cardiac blood vessels. This may cause the death of cardiomyocytes, fibroblast and endothelial cells and lead to cardiac hypertrophy, and ultimately heart failure. Although current surgical treatments based on mechanical revascularisation like coronary bypass, grafting, or angioplasty exist, the chances of recurrent heart failure is higher in these patients. Emerging studies show that formation of new capillaries and blood vessels in the ischaemic heart is a promising therapeutical approach to circumvent the occluded blood supply. Unfortunately, the promising results obtained in animal pre-clinical models by delivery of proangiogenic factors, such as vascular endothelial growth factor (VEGF), into the ischaemic heart, have failed to be translated into clinical practice. Therefore, there is an urgent need to refine current approaches of therapeutic angiogenesis in order to improve neovascularization processes in the ischaemic heart. The recent identification from our laboratory of Plasma Membrane Calcium ATPase 4 (PMCA4) as a negative regulator of VEGF-driven angiogenesis has prompted us to hypothesise that targeting PMCA4regulated pro-angiogenic signalling pathways can be used to increase cardiac revascularisation and restore blood flow to the myocardium at risk.

Methods As a first attempt to elucidate the pro-angiogenic signalling pathways regulated by PMCA4, we have evaluated in this work the expression of genes related to Notch signalling in primary endothelial cells lacking PMCA4. Expression of PMCA4 in HUVEC was silenced by transfection with a siRNA specific for human PMCA4 (or non-target siRNA as control) and stimulated with VEGF. RNA isolated from control or PMCA4-silenced cells was used to screen an array of genes related to Notch signalling. Differences in gene expression were further validated by qPCR using TaqMan gene expression assays.

Results Our results demonstrate that siRNA-mediated PMCA4 gene knockdown in primary endothelial cells strongly enhances the VEGF-induced upregulation of DLL1 and Hey1 gene expression. Lack of PMCA4 did not alter the expression of other important Notch ligands involved in angiogenesis regulation such as DLL4, demonstrating the specificity of our data. These data indicate that PMCA4 acts as a negative regulator of the VEGF-induced expression of DLL1 and Hey1.

Conclusion These results suggest that PMCA4 negatively regulates Notch signaling activation in endothelial cells stimulated by the pro-angiogenic factor VEGF.

Conflict of interest None

\section{BS61 PKC $\alpha \alpha$ KNOCK-DOWN ENHANCES INTIMAL CALCIFICATION IN A MURINE MODEL OF ATHEROSCLEROSIS}

${ }^{1}$ Samantha Borland*, ${ }^{1} J u l i a$ Behnsen, ${ }^{1}$ Abimbola Akerele, ${ }^{1}$ Michael J Sherratt, ${ }^{1}$ Keith Brennan, ${ }^{1}$ Cathy Holt, ${ }^{2}$ Sheila Francis, ${ }^{1}$ Ann Canfield. 'University of Manchester; ${ }^{2}$ University of Sheffield

\subsection{6/heartjnl-2019-BCS.221}

Intimal calcification is the formation of mineralised tissue within atherosclerotic lesions and can lead to an increased risk of plaque rupture and mortality in man. Even though experimental data are sparse, it had been suggested that inhibiting protein kinase $\mathrm{C} \alpha(\mathrm{PKC} \alpha)$ may be of therapeutic benefit in atherosclerosis. However, we recently discovered that loss of PKC $\alpha$ increases mineral deposition by vascular smooth muscle cells (VSMCs) in a transforming growth factor- $\beta$ (TGF $\beta$ )-dependent manner in vitro, suggesting that vascular calcification and its devastating consequences could be increased if patients with cardiovascular disease are treated with PKC $\alpha$ inhibitors. This study tests the hypothesis that $\mathrm{PKC} \alpha$ regulates atherosclerosis and intimal calcification in vivo.

Nine to ten week-old male PKC $\alpha$ knock-out (PKC $\alpha-/-)$ mice crossed with ApoE-/- mice (PKC $\alpha$-/-ApoE-/-) were fed a high-fat, high-cholesterol diet (Western diet) for 8, 18 and 28 weeks. Male ApoE-/- mice fed the same diet were used as controls. En face Oil Red $\mathrm{O}$ staining was significantly 